Association for Information Systems

AIS Electronic Library (AISeL)

Wirtschaftsinformatik Proceedings 2003

Wirtschaftsinformatik

September 2003

\title{
Echtzeit-Kundenberatung im Internet durch synchrone Videokommunikation - Pilotprojekt und Evaluation
}

UlfZschuckelt

Technische Universität Dresden, ulf.zschuckelt@rn.inf.tu-dresden.de

Follow this and additional works at: http://aisel.aisnet.org/wi2003

\section{Recommended Citation}

Zschuckelt, Ulf, "Echtzeit-Kundenberatung im Internet durch synchrone Videokommunikation - Pilotprojekt und Evaluation" (2003). Wirtschaftsinformatik Proceedings 2003. 26.

http://aisel.aisnet.org/wi2003/26

This material is brought to you by the Wirtschaftsinformatik at AIS Electronic Library (AISeL). It has been accepted for inclusion in Wirtschaftsinformatik Proceedings 2003 by an authorized administrator of AIS Electronic Library (AISeL). For more information, please contact elibrary@aisnet.org. 
In: Uhr, Wolfgang, Esswein, Werner \& Schoop, Eric (Hg.) 2003. Wirtschaftsinformatik 2003: Medien - Märkte - Mobilität, 2 Bde. Heidelberg: Physica-Verlag

ISBN: 3-7908-0111-9 (Band 1)

ISBN: 3-7908-0116-X (Band 2)

(C) Physica-Verlag Heidelberg 2003 


\title{
Echtzeit-Kundenberatung im Internet durch synchrone Videokommunikation - Pilotprojekt und Evaluation
}

\author{
Ulf Zschuckelt \\ Technische Universität Dresden
}

\begin{abstract}
Zusammenfassung: Bei einem komplexeren Beratungswunsch greift der Kunde erfahrungsgemäß zum Telefonhörer oder sucht ein Ladengeschäft bzw. Servicepunkt auf. Gerade immer größer werdende Informations- und Vertriebssysteme im Internet verlangen nach einer umfassenden und qualifizierten Beratung. Ein vom Bundesministerium für Bildung und Forschung (BMBF) gefördertes Projekt nimmt sich dieser Herausforderung an und versucht eine Echtzeit-Videoberatung über das Internet zu etablieren. Obwohl die Basistechnologie Videokonferenzsysteme für eine solche Beratung schon seit Jahren existiert, begibt sich die Technische Universität Dresden mit dieser Entwicklung weitestgehend auf Neuland. Deshalb gehört neben der Entwicklung auch eine umfangreiche Evaluation der Wirkungsweise auf die Kunden zum Forschungsinhalt.
\end{abstract}

Schlüsselworte: Video-Call-Center, Echtzeitberatung, Servicedienste, Videokonferenz, H.323, Videokommunikation

\section{Einleitung}

Kundenberatung im Internet hat sich neben der reinen Informationsbereitstellung in den letzten Jahren zu einem wichtigen Bestandteil der Geschäftspolitik von Unternehmen entwickelt. Für eine Vielzahl von Geschäftstransaktionen erweisen sich Informations- und Vertriebssysteme auf Internetbasis als äußerst erfolgreich und effektiv. Besonders im Endkundenbereich, wo eine Differenzierung von den Wettbewerbern über den Preis kaum noch möglich ist, lassen sich Marktanteile nur noch über zusätzliche Serviceangebote stabilisieren oder erhöhen. Ein solches Serviceangebot kann eine umfassende Kundenberatung sein.

In diesem Papier wird eine neue Möglichkeit der Kundenberatung im Internet betrachtet, die der synchronen Videokommunikation. Hierbei soll zuerst allgemein auf Kundenberatung im Internet und speziell auf Kundenberatung mittels synchroner Videokommunikation eingegangen werden. Diese spezielle Form der Beratung wird zurzeit in Dresden im Rahmen eines vom Bundesministerium für Bil- 
dung und Forschung (BMBF) geförderten Forschungsprojekts umgesetzt. Alle aus diesem Pilotprojekt gewonnenen Daten und Erkenntnisse werden einer Evaluation unterzogen und sollen zeigen, dass eine solche Beratungsform technisch realisierbar und ein geeignetes Mittel zur Erhöhung von Kundenzufriedenheit und Kundenbindung sein kann.

\section{Kundenberatung im Internet}

Kundenberatung im Internet unterscheidet sich von der traditionellen Beratung durch sein Trägermedium Internet. Gegenstand der folgenden Betrachtung sollen, bedingt durch das Trägermedium Internet, hauptsächlich elektronische Beratungsformen sein. Dies schließt jedoch nicht aus, dass eine Kundenberatung im Internet keine klassische Kundenberatung, wie

- ein persönliches Beratungsgespräch (face-to-face),

- das Versenden von gedruckten Informationsmaterialien oder

- das Anfertigen eines schriftlichen Angebots

auslöst. Vielmehr arbeiten klassische Beratungsformen und InternetBeratungsformen „Hand in Hand“ und ergänzen sich gegenseitig.

In der Fachliteratur existieren viele unterschiedliche Bezeichnungen von Beratungsformen. Zur Einordnung der Internet-Kundenberatung sollen in Tabelle 1 die wichtigsten Bezeichnungen erklärt und mit Beispielen hinterlegt werden. Diese Übersicht zeigt, dass sich drei Beschreibungsmerkmale für Beratungsformen mit jeweils zwei Eigenschaften finden lassen:

1. Beratungsmedium und Hilfsmittel (klassisch/elektronisch)

2. Beratungsraum (real/virtuell)

3. Kommunikationsbeziehung (synchron/asynchron)

Folglich werden Internet-Beratungen als virtuelle und elektronische Beratungen bezeichnet, die auf asynchroner und/oder synchroner Kommunikation zwischen dem Kunden und Berater basieren können.

\begin{tabular}{|l|l|}
\hline klassisch & $\begin{array}{l}\text { Als klassische Beratung wird die Kundenberatung angesehen, welche } \\
\text { primär nicht auf elektronischen Medien oder Hilfsmitteln basiert. (Bei- } \\
\text { spiele: persönliches Beratungsgespräch, Versenden gedruckter Informati- } \\
\text { onsmaterialen) }\end{array}$ \\
\hline elektronisch & $\begin{array}{l}\text { Die elektronische Beratung basiert auf elektronischen Medien oder } \\
\text { Hilfsmitteln. (Beispiele: Telefongespräch, Internet-Beratung) }\end{array}$ \\
\hline
\end{tabular}




\begin{tabular}{|l|l|}
\hline real & $\begin{array}{l}\text { Die in einem körperlich identifizierbaren Raum stattfindende Beratung } \\
\text { wird als real bezeichnet. (Beispiele: Beratungsgespräch im Ladengeschäft } \\
\text { oder Servicepunkt) }\end{array}$ \\
\hline virtuell & $\begin{array}{l}\text { Bei einer virtuellen Beratung ist der Raum der Beratung nicht identifi- } \\
\text { zierbar. Zwischen Kunde und Verkäufer existiert ein Medium in dem die } \\
\text { Beratung stattfindet. (Beispiele: Telefongespräch, E-Mail, Chat) }\end{array}$ \\
\hline asynchron & $\begin{array}{l}\text { Die Kommunikation zwischen Kunde und Verkäufer läuft zeitlich ver- } \\
\text { setzt ab. Es findet keine Interaktion statt. (Beispiele: E-Mail, Versenden } \\
\text { gedruckter Informationsmaterialen) }\end{array}$ \\
\hline synchron & $\begin{array}{l}\text { Die Kommunikation zwischen Kunde und Verkäufer erfolgt interaktiv zur } \\
\text { gleichen Zeit. (Beispiele: persönliches Beratungsgespräch, Telefonge- } \\
\text { spräch, Chat) }\end{array}$ \\
\hline
\end{tabular}

Tabelle 1: Formen der Kundenberatung

\subsection{Herkömmliche Beratungsformen im Internet}

Beratung und Information sind der wichtigste Bestandteil des personenbezogenen Service im Internet. Beratung ist jedoch immer auf den Kundenwunsch ausgerichtete Information und enthält keine allgemeine Werbeinformation. [HüMa99, S. 283ff] teilt die Informationen, die ein Kunde aus der Beratung gewinnt in 3 Kategorien ein:

- On Demand (spezielle Anfrage des Kunden)

- On Stock (vom Verkäufer vorgefertigte Informationen und bei Bedarf abgerufene Informationen)

- On Delivery (vom Verkäufer ohne Anfrage versandte Informationen)

In allen Kategorien ist das am weitesten verbreitete Kommunikationsmittel die $E$ Mail, wobei der elektronische Newsletter als eine abgewandelte Form betracht werden kann, welcher hauptsächlich als On Delivery-Beratungsmittel genutzt wird. Weitergehend können als On Stock-Beratungsmittel sämtliche Informationen (z. B. FAQ-Listen, Trouble Shooting Guides, elektronische Produktkataloge/Prospekte/Dokumentationen) auf der Web-Seite betrachtet werden.

Bei allen bis jetzt genannten Beratungsmitteln besteht ausschließlich eine asynchrone Kommunikationsbeziehung. Einzig die relativ selten genutzte Möglichkeit des Chat wird dem Kriterium On Demand-Beratungsmittel in synchrone Echtzeit-Kommunikation gerecht, wobei die Kommunikation ausschließlich über Text erfolgt. Eine umfassendere Möglichkeit von Echtzeit-Kommunikation, bei der au- 
diovisuell Informationen übertragen werden, ist die synchrone Videokommunikation.

\subsection{Beratung durch synchrone Videokommunikation}

Mit synchroner Videokommunikation im Internet werden im Allgemeinen Schlagwörter wie Videokonferenzen oder Videotelefonie verbunden. Obwohl sich gemeinsam mit der rasanten Verbreitung des Internets als Massenkommunikationsmedium in den letzten 10 Jahren auch Technologien zur synchronen Videokommunikation über das Internet entwickelt haben, wächst die Akzeptanz von Videokonferenzen bzw. Videotelefonie als Kommunikationsmittel nur langsam.

Von Banken und Finanzdienstleistern wurden in den vergangenen Jahren einige Versuche mit Kundenberatung über so genannte Videochats gestartet, die jedoch nicht zu einer breitenwirksamen Nutzung führten. Alle bis jetzt genannten Synonyme für synchrone Videokommunikation - Videokonferenz, Videotelefonie oder Videochat - sollen im Zusammenhang mit Kundenberatung mit dem Betriff Videoberatung bezeichnet werden, wobei diese nicht mit den „Videoberatung“ beim Frisör oder Augenoptiker zu vergleichen ist, bei der am Computer die neue Frisur bzw. Bille entworfen wird.

\subsubsection{Spezielle Voraussetzungen}

Neben den allgemeinen Voraussetzungen für das Erbringen von Dienstleistungen im Internet (Rechentechnik mit entsprechender Software und Internet-Zugang) müssen für eine Videoberatung von Kunden oder Interessenten weitere spezielle Voraussetzungen gewährleistet sein. Diese Voraussetzungen sollen in Tabelle 2 in 3 Gruppen unterteilt werden.

Für eine Videoberatung sind umfassende spezielle technische Voraussetzungen notwendig, die in letzter Zeit im zunehmenden Maße durch den aktuellen Stand der Rechentechnik und Netzwerktechnik automatisch gewährleistet sind, was niedriger werdenden Investitionskosten zugute kommt. Aktuelle Rechentechnik ist von seiner Performance weitgehend videotauglich und in der Lage Video- und Audioströme in Echtzeit zu kodieren und dekodieren. So ist im Prinzip ein handelsüblicher Heim-PC für diesen Einsatzzweck ausreichend. Wesentlich kostenintensiver ist der Einsatz qualitativ hochwertiger Video/Audio-Aufnahme- und Wiedergabegeräte und der für die Videoübertragung notwendigen Software. Um eine ausreichende Übertragungsqualität zu sichern, empfiehlt sich die Nutzung eines breitbandigen Internet-Zugangs. Die in Deutschland flächendeckend verfügbare DSL-Technik ist dafür hervorragend geeignet und entwickelt sich zunehmend zum Standard des Internet-Zugangs für Privatpersonen sowie klein- und mittelständige Unternehmen, da sie ohne aufwendige Neuverlegung von Anschlussleitungen am Telefonanschluss installierbar ist. 


\begin{tabular}{|l|ll|}
\hline technisch & $\bullet \quad$ videotaugliche Rechentechnik und Räumlichkeiten \\
& $\bullet \quad$ spezielle Software \\
& $\bullet \quad$ breitbandiger Internet-Zugang \\
\hline personell & $\bullet \quad$ geeignetes Personal \\
& $\bullet \quad$ zusätzliche Schulungen \\
\hline \multirow{2}{*}{ organisatorisch } & $\bullet \quad$ Verfügbarkeitsgewährleistung \\
& $\bullet \quad$ schneller Informationszugang \\
\hline
\end{tabular}

Tabelle 2: Spezielle Voraussetzungen für Videoberatung

Eine bei Kundenberatung nicht zu unterschätzende Faktoren sind die personellen Voraussetzungen. Grundsätzlich gilt, dass für eine gute Kundenberatung ausreichend geschultes und geeignetes Personal zur Verfügung stehen muss. Weil bei einer Videoberatung genau wie bei einem persönlichen Beratungsgespräch ein audiovisueller Kontakt zum Kunden besteht, sind äußere Erscheinung, Verhaltensund Sprechweisen des Beraters äußerst wichtig. Die Videokommunikation über das Internet hat jedoch einige grundlegende Unterschiede im Vergleich zu einem realen Gespräch. Diese Unterschiede resultieren aus dem eingesetzten Kommunikationsmedium Internet, weshalb eine zusätzliche Schulung für Videoberatung erforderlich sein kann. Folgende Fakten sollten bei einer Schulung berücksichtigt werden:

- Bei Übertragung von Daten im Internet treten mehr oder weniger große Übertragungsverzögerungen auf. Schon Verzögerungen von 200 bis 300 Millisekunden erfordern eine gewisse Sprechdisziplin, indem durch genügend große Sprechpausen (mindestens das Zweifache der Verzögerung) dem Gesprächsteilnehmer die Möglichkeit der Interaktion gegeben wird.

- Besonders wenn Übertragungswege im Internet stark belastet sind und somit die zur Verfügung stehende Übertragungskapazität beschränkt ist, treten oft Datenpaketverluste auf. Dadurch gehen Video- und Audio-Informationen verloren, was sich in Form von Ruckeln des Bilds oder Stottern des Tons äußert. Diese Effekte versucht die Videokommunikationssoftware durch Reduzierung der verwendeten Übertragungskapazität auszugleichen. Der Berater kann solchen Effekte wirksam entgegentreten, indem er ruckartige Bewegungen vermeidet und langsam und deutlich spricht und somit eine gewisse Ruhe in das Beratungsgespräch bringt.

Wie bei Kundenberatungen die in Echtzeit stattfinden (z. B. persönliches Beratungsgespräch oder Telefonberatung) gehört eine Verfügbarkeitsgewährleistung zu den wichtigsten organisatorischen Voraussetzungen. Die Wartezeit bis zum 
Beratungsgespräch sollte so kurz wie möglich sein und in Spitzenlastzeiten auf eine sinnvolle Zeit begrenzt werden, um eine Überlastung des Kommunikationssystems zu vermeiden. Normale Lastschwankungen können mit aus Erfahrungswerten gewonnen Erkenntnissen durch eine schwankende Anzahl von Beratungspersonal ausgeglichen werden, wobei es meist ökonomisch nicht vertretbar ist das Beratungssystem personell auf selten auftretende Spitzenbelastungen auszulegen. Voraussetzung für eine gute Verfügbarkeit der Kundenberatung ist eine zügige zielorientierte Abarbeitung der Beratungswünsche, was den schnellen Zugang zu allen dafür notwendigen Informationen durch den Berater bedingt.

Damit alle Voraussetzungen gewährleistet sind, müssen die notwendigen finanziellen Mittel zur Verfügung stehen, wobei die Investitions- und Betriebskosten zur Schaffung der technischen Voraussetzungen gut kalkulierbar und im Vergleich zu den Personalkosten relativ gering sind.

\subsubsection{Vergleich mit herkömmlichen Beratungsformen im Internet}

Die Videoberatung ist auf der Suche ein persönliches Beratungsgespräch der realen Welt in einer virtuellen Welt abzubilden und somit die räumliche Distanz zwischen Kunden und Berater zu überbrücken, indem das Internet als Übertragungsmedium genutzt wird. Ein ähnlicher Ansatz ist die Kundenberatung mittels Telefon, bei der das Übertragungsmedium das öffentliche Telefonnetz ist. Bei einem Telefongespräch macht sich jedoch das Fehlen der visuellen Kommunikationskomponente bemerkbar. Mit dieser Komponente sind Gestik, Mimik, Blickkontakte oder auch in ihrer Gesamtheit die Körpersprache verbunden, welche insgesamt einen großen Anteil zur Kommunikation beitragen [PiTe01]. Das Fehlen der visuellen Kommunikationskomponente initiiert bei vielen Kunden jedoch das Gefühl des „schlecht Beraten sein“, wodurch sie eine persönliche Beratung z. B. in einem Ladengeschäft vorziehen, auch wenn dies oft den höheren Zeitaufwand einer Anreise bedeutet.

Zum Vergleich der Videoberatung mit herkömmlichen Beratungsformen im Internet werden ausgewählte Kriterien herangezogen. Die Tabelle 3 zeigt den Vergleich mit den Beratungsformen Web-Seiten (Informationen), E-Mail bzw. Newsletter und Chat.

Bezüglich der Beratungsmöglichkeiten sind Web-Seiten und E-Mail vielfältig einsetzbar. Eine synchrone Echtzeitkommunikation ist mit ihnen jedoch nicht möglich, sodass bei speziellen Beratungsbedürfnissen die Beratungsdauer sehr schnell stark ansteigt. Dies resultiert aus der Tatsache, dass Rückfragen nicht oder nur mit sehr hohem Zeitaufwand und in schriftlicher Form möglich sind. Beim Chat sind sofortige Rückfragen möglich, dessen schriftliche Eingabe aber mehr Zeit in Anspruch nimmt als die akustische Rückfrage einer Videoberatung. 


\begin{tabular}{|l|l|l|l|l|}
\hline Kriterium & Web & E-Mail & Chat & Video \\
\hline on demand - Beratung & nein & ja & ja & ja \\
\hline on stock - Beratung & ja & ja & nein & nein \\
\hline on delivery - Beratung & ja & ja & nein & nein \\
\hline Kommunikationsbeziehung & - & asynchron & synchron & synchron \\
\hline Kommunikationsart & - & schriftlich & schriftlich & audiovisuell \\
\hline Beratungsdauer & hoch & sehr hoch & niedrig & sehr niedrig \\
\hline technische Voraussetzungen & niedrig & niedrig & niedrig & hoch \\
\hline Softwareinstallationsaufwand & niedrig & mittel & mittel & hoch \\
\hline
\end{tabular}

Tabelle 3: Vergleich Videoberatung mit herkömmlichen Beratungsformen im Internet

\section{Pilotprojekt Videoberatung}

Im Rahmen des durch das Bundesministerium für Bildung und Forschung (BMBF) geförderten Forschungsprojekts „,intermobil Region Dresden“ [Inte03] soll auch für den Bereich des Internets eine Möglichkeit gefunden werden, Endkundenberatung mittels Video zu etablieren. Diese Echtzeitvideoberatung wird durch Videokonferenzsysteme nach dem ITU-Standard H.323 [ITU99; Scha96, S. 139ff] realisiert und ist Servicebestandteil eines umfassenden Internetinformations- und Vertriebssystems. Das im Projekt entwickelte Informations-, Vertriebsund Servicesystem wird aufgrund seiner Bindung an den öffentlichen Personenverkehr (ÖPV) als „Virtuelles Mobilitätssystem“ bezeichnet [Schi ${ }^{+} 01$, S. 256].

\subsection{Servicedienstleistung Videoberatung}

Die Informationsfunktion ist schon seit geraumer Zeit in Form eines InternetPortals realisiert, welches auch im Rahmen des Gesamtprojekts weiterentwickelt wird [DORI03]. Eigentliche Neuerung in diesem Teilprojekt ist jedoch die Integration von Vertriebs- und Servicesystemen in das Informationssystem. Damit soll ein einzigartiges geschlossenes System erzeugt werden, welche dem Nutzer des ÖPV erlaubt, alle für die Verwirklichung seines Mobilitätswunsches notwendigen Aktionen auf Basis des Internets durchführen zu können. Als Beispiele dafür lassen sich nennen: 
- Streckenplanung

- Fahrplanauskunft

- Ticketverkauf

- Videoberatung

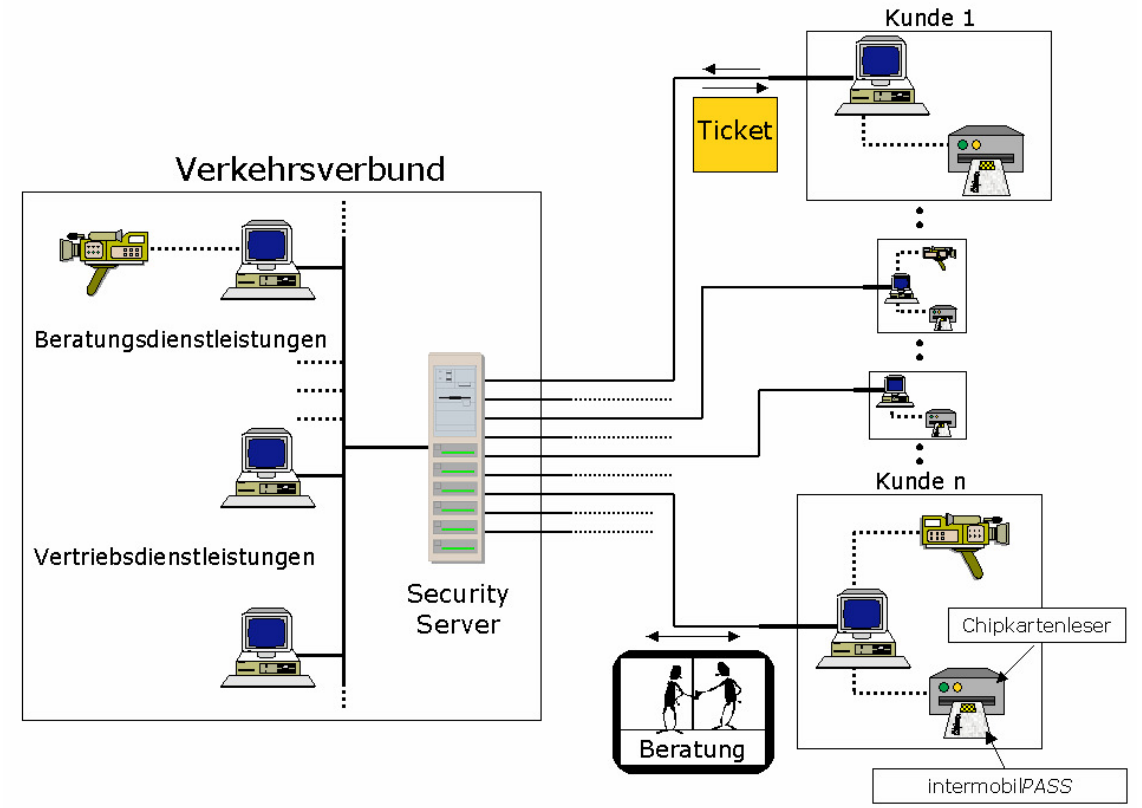

Abbildung 1: Systemtechnische Realisierung von Vertriebs- und Servicedienstleistungen $\left[\mathrm{Schi}^{+} 01\right.$, S. 256]

Während die Streckenplanung und Fahrplanauskunft Teile des Informationssystems sind, werden der Ticketverkauf und die Videoberatung dem Vertriebs- bzw. Servicesystem zugeordnet und auch im „Virtuellen Mobilitätssystem“ verwirklicht (siehe Abbildung 1). Endsysteme für die Nutzer sind im öffentlichen Bereich so genannte Multimediaterminals (im folgenden Nutzerterminals genannt) und im privaten Bereich PCs, wobei alle diese Systeme als Nutzerinterface eine WebUmgebung verwenden. Über die Nutzerterminals wird auch der Vertrieb der elektronischen Tickets realisiert. Die Servicefunktion des Systems wird durch die Videoberatung abgebildet, welche die Nutzer bei Fragen in Zusammenhang mit dem Informations- und Vertriebssystem unterstützen soll [Schi ${ }^{+} 01, \mathrm{~S} .254 \mathrm{ff}$ ]. 


\subsection{Realisierung eines Video-Call-Centers}

Das Konzept für die Realisierung einer Videoberatung auf Web-Basis beinhaltet im Falle des Projekts die Implementierung eines Video-Call-Centers. Prinzipiell ist diese mit einer herkömmlichen Telefon-Call-Center-Lösung vergleichbar, da auch bei der Videoberatung das System einige grundlegende Funktionalitäten bieten muss:

- Bei Überbelegung von Beraterterminals muss eine Warteschlange aufgebaut werden.

- Für das System muss eine Steuerungskomponente existieren, welche die $\mathrm{Zu}$ weisung von Anrufen nach Kriterien wie Priorität, Uhrzeit oder Ort auf bestimmte Beraterterminals zulässt.

- Für die Abrechung und das Controlling müssen die Anrufe protokolliert werden.

Aufgrund des Trägermediums sind bei einer Video-Call-Center-Lösung internetspezifische Anforderungen an das System gestellt worden:

- Das System benötigt besondere Schutzmechanismen gegenüber Manipulationen durch die Nutzer.

- An der Beratung können sich Nutzer mit den unterschiedlichsten Endgeräten nach ITU-Standard H.323 beteiligen. Es existiert dadurch eine Vielzahl von Konfigurationsmöglichkeiten, die berücksichtigt werden müssen.

- Die Beratung soll Teil eines Informations- und Vertriebssystems sein und muss daher in die Prozess-, Funktions- und Datenstrukturen dieser Systeme integriert werden.

Das Konzept eines Video-Call-Centers wird seit Anfang 2001 durch eine Systemimplementierung umgesetzt, welches im November 2002 im Pilotbetrieb beim Verkehrsverbund Oberelbe (VVO) mündete. Die Abbildung 2 zeigt den schematischen Aufbau des Systems zu Beginn des Pilotbetriebs. Das Videoberatungssystem wird in drei Subsysteme mit folgenden Funktionen unterteilt:

- Nutzerterminals: Einzige Funktion der Nutzerterminals ist die Darstellung der Oberfläche des Videoberatungssystems. Sie stellt die Verbindung zu eventuell notwendiger Hardware (z. B. Kamera, Mikrofon und Tastatur) her und gewährleistet die Interaktion mit dem Web-Server. Nutzerinterface ist ein Web-Browser.

- Beraterterminals: Die Beraterterminals haben die gleichen Grundfunktionen wie die der Nutzerterminals. Darüber hinaus besitzen sie zusätzliche Informations- und Administrationsfunktionen zur Steuerung des Videoberatungssystems. Es werden z. B. Informationen über den aktuellen Zustand des Systems angezeigt. Dazu zählen u. a. der Zustand der Warteschlange, Informationen 
über den Anrufer und dessen Aktivitäten im Informations- und Vertriebssystem.

- Managementsystem: Das Managementsystem organisiert die gesamte Kommunikation zwischen Nutzer- und Beraterterminals. Die enthaltenen Komponenten (Applikations-, Web- und Datenbankserver) reagieren hierbei auf die Anfragen der Terminals. Der Applikations-Server beinhaltet sämtliche Anwendungen, die über das Nutzerinterface der Terminals aufgerufen werden. Die Inhalte für das Interface der Terminals werden vom Web-Server bereitgestellt. Über den Web-Server erfolgt auch die Ansteuerung der Anwendungen des Applikations-Servers. Sämtliche Datenbestände wie Konfigurationsdaten der Terminals, Zugriffsdaten und Warteschlangendaten werden im DatenbankServer verwaltet.

Auf weitere technische Details wie z. B. die umfangreichen Schutzmaßnamen gegen Manipulationen des Systems durch Angreifer aus dem Internet soll an dieser Stelle nicht eingegangen werden. Dazu sei auf weitere Ausführungen in [Zsch $\left.{ }^{+} 02\right]$ verwiesen.

Bevor der Pilotbetrieb begonnen werden konnte, waren umfangreiche Laborversuche mit den unterschiedlichsten Videokonferenzsystemen notwendig. Schon hier stellte sich heraus, dass die Standardkonformität der Systeme als äußerst mangelhaft einzuschätzen ist. Trotz dieses Problems wurde das System so implementiert, dass theoretisch jedes Videokonferenzsystem integriert werden kann, welches in einer Web-Umgebung darstellbar ist und definierte Programmierschnittstellen besitzt. Für den Pilotbetrieb wurde das Software-Videokonfernzsystem VidConference der Firma Vidsoft [Vids03] eingesetzt. 


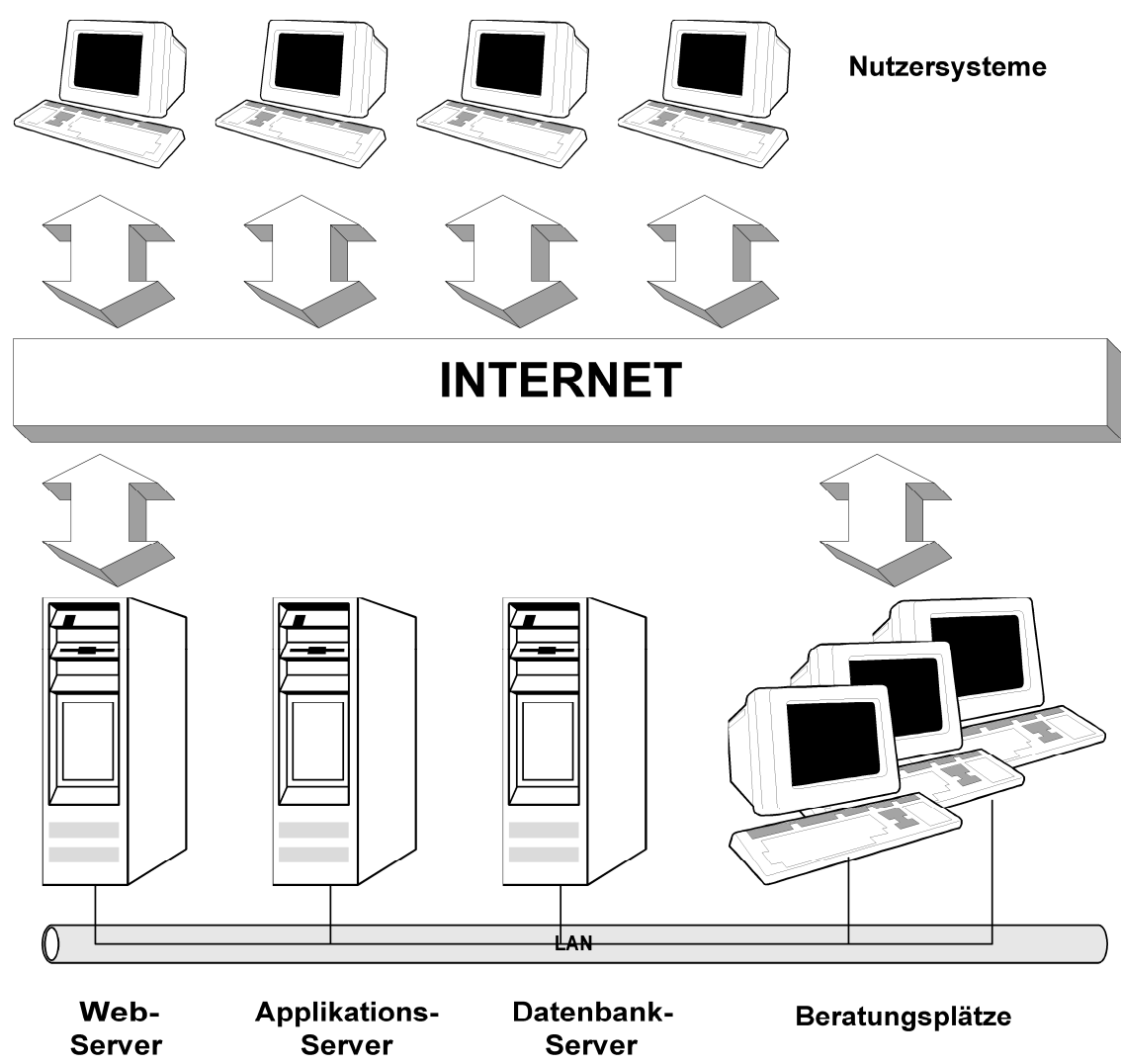

Abbildung 2: Schematischer Aufbau des Videoberatungssystems

Die Auswahl der verwendeten Videokonferenzsoftware stand hauptsächlich unter den Prämissen der zur Verfügung stehenden Nutzerterminals und deren Art der Internet-Anbindung. Die eingesetzten Terminals für den öffentlichen Raum (siehe Abbildung 3) sind mit stark miniaturisierter Rechentechnik ausgestattet, um ein entsprechend schlankes Gehäusedesign garantieren zu können, wodurch in dem meisten Fällen der Einbau eines Hardware-Videokonferenzsystems unmöglich war. Weiterhin mussten besonders kostengünstige Internet-Zugangsalternativen wie T-DSL-Anschlüsse der Deutschen Telekom AG berücksichtigt werden, um die spätere Einbindungsoption privater PCs in die Videoberatung zu berücksichtigen. Für Internet-Zugänge mit asymmetrischen Datenübertragungsraten, wie beim ADSL-Anschluss T-DSL, wurde eine Beratungsvariante entwickelt, bei der kein Video vom Nutzer zum Berater übertragen wird, was keine Auswirkung auf die Beratungsqualität aus Sicht des Nutzers hat. 


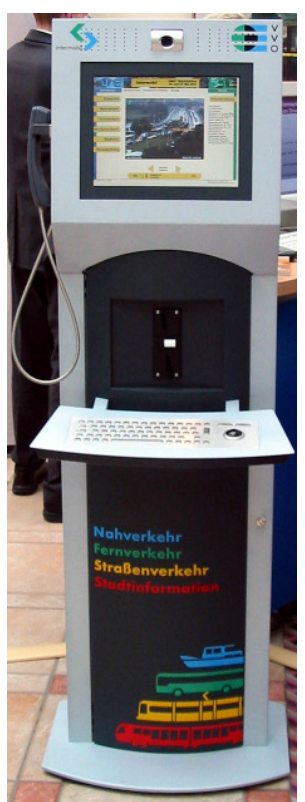

Abbildung 3: Öffentliches Nutzerterminal

Mit dem Start des Videoberatungssystems standen dem Nutzer 4 öffentliche Terminals zur Verfügung, welche von einem Beraterterminal bedient werden. Weil sich die praktische Funktionsfähigkeit des Gesamtsystems erst bei dem Einsatz von mindestens 2 Beraterterminals herausstellen wird, werden 2003 ein weiteres Beraterterminal und 10 zusätzliche Nutzerterminals installiert. Bis Ende 2003 ist geplant in der Region Dresden über 200 solcher Terminals zu installieren, wobei die Anzahl der Beraterterminals an den aktuellen Bedarf angepasst wird.

Um den Dienst Videoberatung erfolgreich etablieren zu können, wird er auch in Zukunft kostenloser Bestandteil des Informations- und Vertriebssystem der öffentlichen Verkehrsbetriebe bleiben. Als kritische Erfolgsfaktoren der Videoberatung lassen sich identifizieren:

- Stabilität: Verfügbarkeit und Übertragungsqualität (technische Stabilität)

- Nutzerfreundlichkeit: intuitive Bedienbarkeit

- Beratungsqualität: schnelle zielorientierte Beratung

Diese kritischen Erfolgsfaktoren werden während des Pilotbetriebs einer permanenten Evaluation unterzogen. 


\section{Evaluation des Pilotprojekt Videoberatung}

Mit der Videoberatung auf Internet-Basis wird absolutes Neuland beschritten. Es existieren praktisch keine Erfahrungen mit dem Betrieb eines Video-Call-Centers. Daher ist eine permanente Evaluation während des Pilotbetriebs unumgänglich, um die Auswirkung selbst kleinster Veränderungen feststellen und die im vorangegangen Kapitel genannten kritischen Erfolgsfaktoren Stabilität, Nutzerfreundlichkeit und Beratungsqualität sichern zu können.

Für eine umfassende Evaluierung bedarf es quantitativer und qualitativer Messungen am Videoberatungssystem.

\subsection{Quantitative Messungen}

Die quantitativen Messungen erfolgen durch die Protokollierung sämtlicher Zeitparameter der Beratungsgespräche, wie:

- Rufzeitpunkt (gleichzeitig Einreihung in die Warteschlange)

- Zuweisungszeitpunkt zu einem Beraterterminal

- Beratungsbeginn

- Beratungsende

Diese in Echtzeit vorliegenden Daten können zu einer sofortigen quantitativen Bewertung herangezogen werden. Weil neben den Zeitdaten auch die Identitäten der jeweils beteiligten Nutzer- bzw. Beraterterminals gespeichert werden, sind folgende grundsätzliche Auswertungen möglich:

- Auslastung der Nutzer- und Beraterterminals

- Wartezeit in der Warteschlange

- Gesprächsdauer

Neben diesen Erfolgsmessungen werden jedoch auch Fehlermessungen durchführt. Zu den fehlerhaften Beratungen zählen alle Beratungen, bei denen sich die Beratungsdauer nicht ermitteln lässt. Schon während des Anwahlversuchs zeichnet das System auftretende Fehler in Form eines Fehlercodes auf. Aus diesen Aufzeichnungen lassen sich bereits Rückschlüsse auf die Qualität des Videoberatungssystems ziehen.

\subsection{Qualitative Messungen}

Ergänzend zu quantitativen Messungen erfolgt als Grundlage einer qualitativen Bewertung des Videoberatungssystems die Aufzeichnung sämtlicher Konfigurati- 
onsparameter der am Beratungsgespräch beteiligten Terminals. Die Aufzeichnung dieser technischen Parameter steht im direkten Zusammenhang mit einer subjektiven Bewertung jedes Beratungsgespräches durch den Berater. Dieser muss am Ende jedes Beratungsgesprächs folgende Fragen beantworten:

- War die Beratung erfolgreich?

- War der Kunde mit der Beratung zufrieden?

- Hatten Sie alle für die Beratung notwendigen Informationen zur Verfügung?

- Hatten Sie oder der Kunde Verständigungsprobleme?

- Hatte der Kunde Bedienungsprobleme?

\subsection{Bewertung der Messergebnisse}

Die Bewertung der Messergebnisse erweist sich als sehr komplex, weil die Qualität der Beratung von vielen Einflussfaktoren abhängig ist, die nicht im direkten Zusammenhang mit der verwendeten Technologie „Video über Internet“ steht. Eine Einteilung in z. B. technologieunabhängige und technologieabhängige Bewertungen erweist sich als fast unmöglich, weil Beratungsquantität und -qualität sehr stark an die Qualität der technischen Hilfsmittel gebunden ist. Trotzdem muss es möglich sein einen Beratungsmisserfolg auf einen menschlichen Fehler oder einen technischen Fehler zurückführen zu können. Schon ein einfacher Bedienungsfehler an der eingesetzten Technik zeigt jedoch, dass eine eindeutige Fehlerzuordnung dem Mensch oder der Technik schwierig ist. Solche schwierigen Bewertungen sind nur über längerfristige quantitative und qualitative Messungen möglich. Erst danach kann erkannt werden, ob der Mensch oder die Technik Ursache eines Beratungsmisserfolgs ist.

Entsprechend den Messungen wird auch bei den Bewertungsmethoden in einen quantitativen und einen qualitativen Teil unterschieden. Welche Bewertungen aus ausgewählten quantitativen Parametern abgeleitet werden können, zeigt folgende Tabelle:

\begin{tabular}{|l|l|}
\hline Parameter & Bewertung \\
\hline Auslastung & $\begin{array}{l}\text { Anhand der Auslastung des Beratungssystems kann die Akzeptanz } \\
\text { des Beratungssystems abgeleitet werden. Es gibt jedoch vielfältige } \\
\text { Ursachen für eine niedrige Auslastung, z. B.: }\end{array}$ \\
& $\begin{array}{l}\text { Der Zugang für die Beratung ist erschwert oder unbekannt, } \\
\text { weil Nutzerterminals falsch positioniert oder nicht als solche } \\
\text { erkennbar sind. }\end{array}$ \\
\hline Die Qualität der Beratung ist schlecht.
\end{tabular}




\begin{tabular}{|l|l|}
\hline & $\begin{array}{l}\text { — Die Bedienbarkeit des Nutzterminals ist nicht gewährleistet. } \\
\text { • Das Beratungssystem ist überdimensioniert. }\end{array}$ \\
\hline Vermittlungsquote & $\begin{array}{l}\text { Die niedrige Vermittlungsquote von Beratungsgesprächen ist meist } \\
\text { auf technische Probleme oder auf eine Überfüllung der Warte- } \\
\text { schlange zurückzuführen. }\end{array}$ \\
\hline Beratungsdauer & $\begin{array}{l}\text { Die Beratungsdauer hat einen hohen Einfluss auf die Verfügbarkeit } \\
\text { des Beratungssystems. Von der Dauer selber sich lassen sich je- } \\
\text { doch keine Rückschlüsse auf die Qualität der Beratung ziehen. }\end{array}$ \\
\hline
\end{tabular}

Tabelle 4: Quantitative Bewertungsparameter

Offensichtlich lassen sich aus den quantitativen Bewertungsparametern Auslastung und Beratungsdauer ohne eine gleichzeitige Betrachtung der qualitativen Bewertungsparameter (siehe Tabelle 5) nur schlecht Rückschlüsse auf die Ursachen der gemessenen Werte ziehen.

\begin{tabular}{|l|l|}
\hline Parameter & Bewertung \\
\hline Erfolg & $\begin{array}{l}\text { Der Beratungserfolg stellte den reinen Fakt der Zielerreichung dar und } \\
\text { trifft noch keine Aussage über die Qualität der Beratung. }\end{array}$ \\
\hline Zufriedenheit & Die Zufriedenheit ist der Qualitätsparameter des Beratungserfolgs. \\
\hline Verständigung & $\begin{array}{l}\text { Ursache einer mangelhaften Verständigung sind technische Übertra- } \\
\text { gungsprobleme. Die Verständigung ist Voraussetzung für den Erfolg } \\
\text { und die Zufriedenheit. }\end{array}$ \\
\hline Bedienbarkeit & $\begin{array}{l}\text { Zwingende Voraussetzung für ein erfolgreiches Beratungsgespräch ist } \\
\text { die Bedienbarkeit des Systems. }\end{array}$ \\
\hline
\end{tabular}

Tabelle 5: Qualitative Bewertungsparameter

Bei der gemeinsamen Betrachtung aller Bewertungsparameter lassen sich folgende Regeln, die auch als Handlungsanweisung für Systemverbesserung geeignet sind, aufstellen:

1. Ziel der Beratung ist der Beratungserfolg und eine möglichst hohe Vermittlungsquote.

2. Der Beratungserfolg tritt nur dann ein, wenn die Bedienbarkeit des Systems gewährleistet ist.

3. Die Beratungsdauer hat direkten Einfluss auf die Auslastung des Systems. 
4. Bei mangelnder Verständigung steigt die Beratungsdauer und sinkt die Zufriedenheit des Nutzers.

Aus ökonomischer Sicht muss ein Optimum aus Beratungsdauer und Zufriedenheit des Nutzers gefunden werden. Dieses Optimum kann je nach Beratungsgegenstand und Nutzergruppe an einem anderen Punkt liegen. Im Fall der Videoberatung für den ÖPV wird der Kunde mit einer kurzen Beratung zufrieden sein. Es kann im Normalfall von relativ einfachen Beratungswünschen mit niedriger Komplexität ausgegangen werden.

\section{Zusammenfassung und Ausblick}

Die Videoberatung ist eine Ergänzung zu den klassischen Beratungsformen im Internet, welche oft auf Web-Seiten, E-Mail oder Chat basieren. Synchrone audiovisuelle Kommunikation stellt relativ hohe Ansprüche an die eingesetzte Hardund Software bzw. Netzanbindung. Der für eine Videoberatung notwendige technische, personelle und organisatorische Aufwand wird sich daher grundsätzlich vom Aufwand klassischer Beratungsformen unterscheiden. Ein höherer Aufwand für eine Videoberatung bedeutet nicht zwangsläufig eine Unrentabilität, wenn z. B. durch die neue Beratungsform klassische, weitaus unrentablere Beratungsformen abgelöst werden können.

Das Pilotprojekt Videoberatung soll zeigen, dass Kundenberatung im Internet durch synchrone Videokommunikation keine Utopie mehr ist und sich aus technischer Sicht realisieren lässt. Mit der Videoberatung ist es dem Kunden endlich möglich, audiovisuellen Kontakt zu einem Berater über das Internet herzustellen. Im Vergleich zu herkömmlichen Beratungsformen im Internet besteht die Möglichkeit, wesentlich schneller komplexere Beratungsvorgänge handhaben zu können.

Wie erste Beobachtungen ergeben, ist die Beratung über ein Video-Call-Center für den Kunden sehr ungewohnt. Bis zum Projektende Mitte 2004 ist es daher Ziel, den Kunden an diese neue Beratungsform zu gewöhnen. Das Ziel ist dann erreicht, wenn der Kunde die Videoberatung als Alternative zu einer persönlichen Beratung oder einer Telefonberatung akzeptiert. Ist diese Akzeptanz vorhanden, kann an eine schrittweise Ablösung klassischer Call-Center-Lösungen gedacht werden. Insgesamt ist die Videoberatung eine perfekte Ergänzung zu bereits vorhandenen Servicedienstleistungen und die erste wirkliche Möglichkeit einer EchtzeitKundenberatung im Internet. 


\section{Literatur}

[DORI03] o. V.: DORIS - Stadt- und Verkehrsinformationssystem für die Region Dresden. http://www.intermobil.org. Abruf am 2003-01-25.

[HüMa99] Hünerberg, R.; Mann, A.: Online-Service. In Bliemel, F.; Fassott, G.; Theobald, A. (Hrsg.), Electronic Commerce: Herausforderung-Anwendung-Perspektive. Gabler: Wiesbaden, 1999, S. 279-297.

[Inte03] 0. V.: intermobil Region Dresden. Zukünftige Strategien zur Mobilitätssicherung in mittleren Ballungsräumen; Forschung - Entwicklung - Realisierung. http://www.intermobil-dresden.de. Abruf am 2003-01-24.

[ITU99] o. V.: ITU-T Recommendations on CD-ROM. International Telecommunication Union, Geneva, 1999.

[PiTe01] o. V.: Hintergrundinformationen zur Videokommunikation. PictureTel VideocomInfo Center. http://www.bachert.de/video/info/backgrnd.htm, 2001. Abruf am 2003-0124.

[Scha96] Schaphorst, R.: Videoconferencing and videotelephony: technology and standards; Artech House Inc., Nordwood, 1996.

[Schi $\left.{ }^{+} 01\right]$ Schill, A., Braun, I., Zschuckelt, U.: Virtuelle Mobilitätsdienste: Allgemeine Konzepte und Pilotvorhaben in der Region Dresden; 18. Verkehrswissenschaftliche Tage Dresden - Verkehr und Mobilität in der Informationsgesellschaft; Dresden, September 2001, S. 250-261.

[Vids03] o. V.: Vidsoft GmbH. http://www.vidsoft.de. Abruf am 2003-01-27.

[Zsch $\left.{ }^{+} 02\right]$ Zschuckelt, U., Braun, I., Schill, A.: Video-based Customer Consulting via Internet using videoconferencing standard H.323; Information Systems: The e-Business Challenge, Edited by Roland Traunmüller, Kluwer Academic Publischer, Boston/Dordrecht/London, 2002, S. 147-155. 